OPEN ACCESS

Edited by:

Chin-Hsiao Tseng,

National Taiwan University, Taiwan

Reviewed by:

Ken Muramatsu,

Kyorin University, Japan

Maria Pia Giannoccaro,

University of Bologna, Italy

*Correspondence:

Lingling Xu

lucyling/@126.com

${ }^{\dagger}$ These authors have contributed equally to this work

Specialty section:

This article was submitted to

Clinical Diabetes,

a section of the journal

Frontiers in Endocrinology

Received: 13 May 2021

Accepted: 31 August 2021 Published: 21 September 2021

Citation:

Lin X, Chen C, Liu Y, Peng Y, Chen Z, Huang $H$ and $X u L$ (2021) Peripheral Nerve Conduction And Sympathetic Skin Response Are Reliable Methods to Detect Diabetic Cardiac Autonomic Neuropathy.

Front. Endocrinol. 12:709114. doi: 10.3389/fendo.2021.709114

\section{Peripheral Nerve Conduction And Sympathetic Skin Response Are Reliable Methods to Detect Diabetic Cardiac Autonomic Neuropathy}

\author{
Xiaopu Lin ${ }^{1 \dagger}$, Chuna Chen ${ }^{2,3+}$, Yingshan Liu ${ }^{2,3}$, Yu Peng ${ }^{4}$, Zhenguo Chen ${ }^{2,3}$, \\ Haishan Huang ${ }^{2,3}$ and Lingling $\mathrm{Xu}^{2,3 *}$ \\ ${ }^{1}$ Department of Huiqiao Building, Nanfang Hospital, Southern Medical University, Guangzhou, China, ${ }^{2}$ Department of \\ Endocrinology, Shenzhen Hospital, Southern Medical University, Shenzhen, China, ${ }^{3}$ The Third School of Clinical Medicine, \\ Southern Medical University, Guangzhou, China, ${ }^{4}$ Department of Neurology, Nanfang Hospital, Southern Medical University, \\ Guangzhou, China
}

Aim: This study aimed to investigate the role of nerve conduction studies (NCS) and sympathetic skin response (SSR) in evaluating diabetic cardiac autonomic neuropathy (DCAN).

Methods: DCAN was diagnosed using the Ewing test combined with heart rate variability analysis. NCS and SSR were assessed by electrophysiological methods. The association between NCS/SSR and DCAN was assessed via multivariate regression and receiveroperating characteristic analyses.

Results: The amplitude and conduction velocity of the motor/sensory nerve were found to be significantly lower in the DCAN+ group (all $P<0.05$ ). A lower amplitude of peroneal nerve motor fiber was found to be associated with increased odds for DCAN (OR 2.77, $P<0.05)$. The SSR amplitude was lower while the SSR latency was longer in the DCAN+ group than in the DCAN- group. The receiver-operating characteristic analysis revealed that the optimal cutoff points of upper/lower limb amplitude of SSR to indicate DCAN were $1.40 \mathrm{mV}$ (sensitivity, 61.9\%; specificity, 66.3\%, $P<0.001$ ) and $0.85 \mathrm{mV}$ (sensitivity, $66.7 \%$; specificity, $68.5 \%, P<0.001$ ), respectively. The optimal cutoff points of upper/ lower limb latency to indicate DCAN were 1.40 s (sensitivity, 61.9\%; specificity, 62\%, $P<$ 0.05 ) and $1.81 \mathrm{~s}$ (sensitivity, 69.0\%; specificity, 52.2\%, $P<0.05$ ), respectively.

Conclusions: NCS and SSR are reliable methods to detect DCAN. Abnormality in the peroneal nerve (motor nerve) is crucial in predicting DCAN. SSR may help predict DCAN.

Keywords: diabetic cardiac autonomic neuropathy, Ewing test, heart rate variability, nerve conduction, sympathetic skin response, T2DM

\section{INTRODUCTION}

Diabetic cardiac autonomic neuropathy (DCAN) is a serious long-term complication of diabetes mellitus (DM). It results from chronic damage to autonomic nerve fibers that innervate the heart and blood vessels, in turn causing abnormalities in heart rate and vascular dynamics. It significantly increases the morbidity and mortality of patients with diabetes $(1,2)$. Early diagnosis of DCAN may 
reduce the risk of painless myocardial ischemia, myocardial infarction, and sudden cardiac death associated with DCAN (3). Therefore, the American Diabetes Association (ADA) recommends that the clinical screening for DCAN should be routinely performed in patients with diabetes (4).

Screening for DCAN is important because it is a frequently overlooked complication of diabetes, especially in the early stage. The Ewing test and heart rate variability (HRV) analysis are recommended by the ADA as the diagnostic tests for DCAN (5). The Ewing test, which assesses autonomic reflexes by measuring heart rate and blood pressure during activities such as the Valsalva, deep breathing, or standing, is a classic clinical test for cardiac autonomic neuropathy. However, it is a semiquantitative method and relies on patients' cooperation, and its interpretation is operator dependent. HRV analysis provides indirect insight into autonomic nervous system tone and plays a well-established role as a marker of cardiovascular risk (6).

HRV is composed of time-domain and frequency-domain components, but it does not estimate cardiac reflexes $(7,8)$. Some prior studies have demonstrated that the diagnostic specificity can be increased by combining the results of the Ewing test and $\operatorname{HRV}(5,9)$, which was the approach used in this study.

Objective examinations that can identify DCAN during its early stage are critical. Considering that Ewing test and HRV analysis tests are time-consuming and require a high degree of cooperation from patients, it is difficult to screen for every diabetic patient in clinical work. Therefore, it is important to find a more convenient method to screen DCAN. Nerve conduction studies (NCS) and the sympathetic skin response (SSR) are routinely employed in the diagnosis of diabetic peripheral neuropathy $(10,11)$, which may be useful in identifying patients at high risk for DCAN. The present study was designed to investigate the role of NCS and SSR in the evaluation of DCAN in type 2 diabetes mellitus (T2DM).

\section{MATERIALS AND METHODS}

\section{Study Design}

This cross-sectional, open-label, controlled clinical study was performed to explore the role of nerve conduction studies (NCS) and sympathetic skin response (SSR) in evaluating diabetic cardiac autonomic neuropathy (DCAN). Participants were recruited from the inpatients at the Department of Endocrinology of Nanfang Hospital. This study was reviewed and received ethical approval from the Ethics Committee of Nanfang Hospital, Southern Medical University. Written consent was obtained from all study participants at the time of enrollment. The Chinese Clinical Trials Registration Number is ChiCTR1900020491.

The inclusion criteria were as follows: age more than 18 years and current diagnosis of T2DM.

The exclusion criteria were as follows: inability to undertake the examination, neurological or autonomic disorders caused by other diseases (Guillain-Barre syndrome, Shy-Drager syndrome, and so forth), history of coronary artery disease (such as myocardial infarction or angina), arrhythmia, severe heart failure, malignant tumor, limb trauma, chronic rheumatic disease, thyroid function abnormalities, severe psychiatric disorders that interfered with the patient's ability to complete study procedures, severe hepatic or renal dysfunction [GFR < $30 \mathrm{~mL} /\left(\mathrm{min} \cdot 1.73 \mathrm{~m}^{2}\right)$ ], alcohol abuse (women 14 units per week and men 21 units per week in the last year), intake of neurotoxic medications or beta-blockers, vitamin B12 deficiency, and pregnancy.

The participants' clinical data, including sex, age, duration of diabetes, height, body weight, body mass index (BMI), and smoking history, were recorded. Laboratory measurements, including triglyceride (TG), total cholesterol (TC), high-density lipoprotein cholesterol (HDL), low-density lipoprotein cholesterol (LDL), fasting plasma glucose (FPG), postprandial blood glucose (PBG), glycosylated hemoglobin (HbAlc), fasting C-peptide (FCP), and fasting insulin (FINS), were obtained after an 8 -h fast.

\section{Ewing Test and HRV Analysis}

The presence of DCAN was first assessed by measuring the four cardiovascular reflexes as described by Ewing in 1970: heart rate variation with deep breathing with an assessment of expiration to inspiration (E/I) ratio, heart rate analysis in the standing position (the $30 \mathrm{~s} / 15 \mathrm{~s}$ ratio), Valsalva ratio, and blood pressure response to positional changes from lying to standing (orthostatic hypotension, $\mathrm{OH})(12,13)$. Three of these tests assessed the parasympathetic functions, such as E/I ratio, $30 \mathrm{~s} / 15 \mathrm{~s}$ ratio, and Valsalva ratio, while $\mathrm{OH}$ assessed the sympathetic function (14) primarily. An E/I ratio more than the age-specific reference value, a Valsalva ratio $\geq 1.21$, a posture ratio $\geq 1.04$, and a systolic blood pressure reduction in response to the standing of $\leq 10 \mathrm{~mm} \mathrm{Hg}$ were considered normal. An E/I ratio below the ageadjusted values, a Valsalva ratio $\leq 1.10$, a posture ratio $\leq 1.00$, and a systolic blood pressure fall in response to the standing of $\geq 20$ $\mathrm{mm} \mathrm{Hg}$ were considered abnormal. Values that fell between normal and abnormal were considered borderline. Each of these four tests was assigned a score of 0 for normal, 0.5 for borderline, and 1 for abnormal results; the sum of these four scores made up the Ewing score, which was used to assess the severity of DCAN. An Ewing score $\geq 2$ was considered abnormal. Participants were instructed to avoid food and particular pharmacological agents (antidepressants, neuroleptics, nicotine, and caffeine) for $12 \mathrm{~h}$ preceding the examination.

HRV was evaluated according to the European Society of Cardiology guidelines (15) using a 24-h Holter monitor. Timedomain HRV and frequency-domain HRV indexes were analyzed. Time-domain HRV indexes included the standard deviation (SD) of all normal-to-normal (NN) intervals (SDNN), SD of the average $\mathrm{NN}$ intervals calculated over 5-min periods of the entire recording (SDANN), root mean square successive difference in the R-R interval ( $r M S S D, m s$ ), and percentage of adjacent $\mathrm{RR}$ intervals with a difference of duration greater $50 \mathrm{~ms}$ (PNN50, \%). The frequency-domain $\mathrm{HRV}$ indexes included low-frequency power (LF, $\mathrm{ms}^{2}$ ) and high-frequency power $\left(\mathrm{HF}, \mathrm{ms}^{2}\right)$. HRV was considered 
abnormal if at least two of the following six abnormal parameters were met: SDNN $<50 \mathrm{~ms}$, SDANN $<40 \mathrm{~ms}$, PNN50 <0.75\%, rMSSD $<15 \mathrm{~ms}, \mathrm{LF}<300 \mathrm{~ms}^{2}$, and $\mathrm{HF}<300 \mathrm{~ms}^{2}(15,16)$.

In this study, the presence of DCAN $(\mathrm{DCAN}+)$ was defined as having both an abnormal Ewing score $(\geq 2)$ and an abnormal HRV analysis ( $\geq 2$ abnormal parameters).

\section{Electrophysiologic Evaluation}

NCS were performed using the Viking Quest (Nicolet VIASYS Healthcare, USA). Skin temperature was maintained above $32^{\circ} \mathrm{C}$ in the upper limbs and above $31^{\circ} \mathrm{C}$ in the lower limbs. The filtering frequency was $20 \mathrm{~Hz}$ to $10 \mathrm{kHz}$. The compound muscle action potential (CMAP) amplitude, latency, and motor conduction velocity $(\mathrm{CV})$ of the median, ulnar, posterior tibial, and peroneal nerves were recorded. The CMAP amplitude was recorded from peak to peak by a supramaximal stimulation. The supramaximal stimulation was defined as $10 \%$ addition of stimulation charge after CMAP amplitude reaches its maximum. The measurements of sensory nerve action potential (SNAP) amplitude, latency, and sensory conduction velocity $(\mathrm{CV})$ of the median, ulnar, and sural nerves were recorded also by supramaximal stimulation. The mean value of 20 results was taken for the sensory NCS. For each individual, the mean of the motor nerve amplitude was calculated using the following formula: Amplitude of motor nerve $=$ (Amplitude of median nerve $\mathrm{M}+$ Amplitude of ulnar nerve $\mathrm{M}+$ Amplitude of posterior tibial nerve $\mathrm{M}+$ Amplitude of peroneal nerve $\mathrm{M}) / 4$. The means of motor nerve CV, sensory nerve amplitude, and sensory nerve CV were calculated using the same method.

In the present study, diabetic peripheral neuropathy (DPN) was defined as the present of one or more abnormal nerve conduction result (amplitude or CV) in at least two different peripheral nerve (10).

\section{Sympathetic Skin Response}

The SSR was studied using the standard method (17). The room temperature was maintained at $25^{\circ} \mathrm{C}-26^{\circ} \mathrm{C}$. A standard electromyographic active electrode was attached to the right palm and sole and the reference electrode to the dorsum of the hand and foot. The stimulus used was a single electrical stimulus at the right wrist of $10 \mathrm{~mA}$ for $100 \mathrm{~ms}$ duration. This stimulation procedure was standardized in previous studies on fibromyalgia and correlated with symptoms (18). Stimuli were delivered unexpectedly and at random intervals between 30 and $60 \mathrm{~s}$. Five consecutive stimuli were delivered. The latency was measured from the onset of the stimulus artifact to the onset of the first negative deflection and expressed in seconds. The amplitude was measured from the baseline to the maximal negative peak and expressed in $\mathrm{mV}$. The response was considered absent if no consistent voltage change occurred using a sensitivity of $50 \mathrm{mV}$ per division after three trials at maximum stimulus intensity. Response latencies were considered pathological when exceeding two SD more than the mean latency in the control group. The SSR habituation was considered as the percent rate of the maximal amplitude change between the fifth and the first response. A value less than 1 indicated habituation.

\section{Statistical Analysis}

For continuous variables, the results were presented as the mean \pm SD if normally distributed, and median (interquartile range, IQR) if nonnormally distributed. The Student $t$ test (normally distributed data) and the Kruskal-Wallis test (nonparametric data) were employed to examine differences between groups. Categorical data were analyzed using Pearson's $\chi^{2}$ test. Multivariate logistic regression was performed to determine which nerve parameter had the greatest predictive value for the diagnosis of DCAN. Receiver-operating characteristic (ROC) analysis was performed to assess the optimal SSR cutoff for indicating DCAN. A two-sided $P<0.05$ was assumed to be statistically significant, while $P<0.001$ was taken as highly significant. All statistical analyses were performed using SPSS statistics software (version 20.0, 2011; IBM, USA).

\section{RESULTS}

\section{Clinical Characteristics of Participants}

From January 2019 to June 2019, 172 patients with T2DM were screened for inclusion in this study. Of these, 38 patients were excluded based on the listed exclusion criteria, and the remaining 134 participants were enrolled. Further, 42 patients $(31.34 \%)$ were diagnosed with DCAN as described early (both positive Ewing test and positive HRV analysis). The average test results in each group are shown in Table 1. The baseline clinical and laboratory characteristics of the patients are also shown in Table 1. Patients with DCAN were more likely to be male compared with controls $(P<0.05)$, and were older $(P<0.001)$. Participants with DCAN also had a slightly lower FPG $(P<0.05)$. Diabetes duration, smoking, BMI, blood pressure, TG, TC, HDL, LDL, PBG, HbA1c, FCP, and FINS were similar between the two groups.

\section{Ewing Test Parameters Between DCAN+ and DCAN- Groups}

Statistically significant differences were observed in E/I ratio $(P<$ $0.001)$, Valsalva ratio $(P<0.001), 30 \mathrm{~s} / 15 \mathrm{~s}$ ratio $(P<0.001), \mathrm{OH}$ $(P<0.05)$, and Ewing score $(P<0.001)$ between DCAN+ group and DCAN- group (Supplementary Table 1).

\section{HRV Parameters Between DCAN+ and DCAN- Groups}

Statistically significant differences were found in SDANN $(P<$ $0.05), \mathrm{LF}(P<0.001)$, and HF $(P<0.001)$ between DCAN+ and DCAN- groups, while SDNN, rMSSD, and pNN50 were similar between the two groups (Supplementary Table 2).

\section{NCS Parameters Between DCAN+ and DCAN- Groups}

There are $78.57 \%$ (33/42) patients diagnosed as DPN (based on NCS test) in DCAN group, while 59.78\% (55/92) in non-DCAN group $(P<0.05)$. The averaged NCS results of different motor and sensory nerves were compared in Table 2. The amplitude (7.96 \pm $2.66 \mathrm{mV}$ vs. $9.60 \pm 2.22 \mathrm{mV}, P<0.001)$ and $\mathrm{CV}(44.22 \pm 5.10 \mathrm{~m} / \mathrm{s}$ vs. 
TABLE 1 | Clinical characteristics of patients with T2DM and DCAN versus those without DCAN.

\begin{tabular}{|c|c|c|c|}
\hline & $\mathrm{DCAN}+(n=42)$ & DCAN- $(n=92)$ & $P$ \\
\hline $\operatorname{Sex}(M / F)$ & $30 / 12$ & $48 / 44$ & $<0.05^{\star}$ \\
\hline Age (year) & $63.86 \pm 10.57$ & $55.35 \pm 12.63$ & $<0.001^{* *}$ \\
\hline Duration (year) & $10.13 \pm 7.59$ & $8.25 \pm 6.79$ & n.s. \\
\hline Smoking (\%) & $26.5 \%$ & $32.5 \%$ & n.s. \\
\hline $\mathrm{BMI}\left(\mathrm{kg} / \mathrm{m}^{2}\right)$ & $24.22 \pm 3.51$ & $23.20 \pm 3.09$ & n.s. \\
\hline SBP $(\mathrm{mm} \mathrm{Hg})$ & $148.38 \pm 26.73$ & $140.07 \pm 24.11$ & n.s. \\
\hline DBP (mm Hg) & $81.95 \pm 12.66$ & $82.70 \pm 11.45$ & n.s. \\
\hline TG (mmol/L) & $2.08 \pm 1.83$ & $1.82 \pm 1.27$ & n.s. \\
\hline TC (mmol/L) & $4.55 \pm 1.83$ & $4.95 \pm 1.57$ & n.s. \\
\hline HDL (mmol/L) & $0.97 \pm 0.29$ & $1.03 \pm 0.28$ & n.s. \\
\hline LDL (mmol/L) & $2.85 \pm 1.28$ & $3.08 \pm 0.99$ & n.s. \\
\hline FPG (mmol/L) & $8.45 \pm 2.98$ & $8.66 \pm 3.34$ & $<0.05^{\star}$ \\
\hline PBG (mmol/L) & $15.27 \pm 7.30$ & $14.97 \pm 5.63$ & n.s. \\
\hline HbA1c(\%) & $8.83 \pm 2.74$ & $9.62 \pm 2.65$ & n.s. \\
\hline FCP (ng/mL) & $2.96(1.07-3.69)$ & 1.69 (1.02-2.73) & n.s. \\
\hline FINS $(\mu \mathrm{U} / \mathrm{mL})$ & $8.81(3.60-15.44)$ & $6.69(3.49-13.79)$ & n.s. \\
\hline
\end{tabular}

Values were expressed as mean \pm SD for normally distributed data and median with interquartile range for nonnormally distributed data, or $n$ (\%). Differences between the groups were

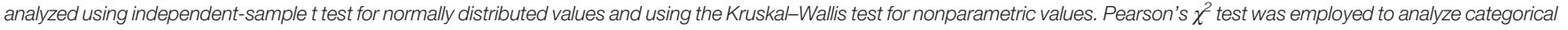

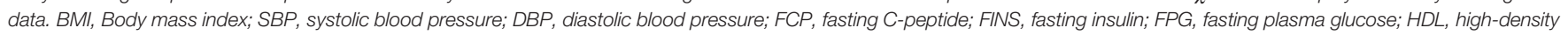
lipoprotein; LDL, low-density lipoprotein; PBG, postprandial blood glucose; TG, triglycerides; TC, total cholesterol.

${ }^{\star} P<0.05,{ }^{* *} P<0.001$.

n.s., Not significant.

$46.62 \pm 3.96 \mathrm{~m} / \mathrm{s}, P<0.05)$ of the motor nerve were lower in the DCAN+ group than in the DCAN- group. Similarly, the sensory amplitude $(17.90 \pm 13.66 \mathrm{mV}$ vs. $26.24 \pm 11.92 \mathrm{mV}, P<0.001)$ and CV $(48.13 \pm 7.27 \mathrm{~m} / \mathrm{s}$ vs. $51.16 \pm 6.23 \mathrm{~m} / \mathrm{s}, P<0.05)$ were significantly lower in the DCAN+ group than in the DCANgroup (Table 2 ).

\section{Nerve Conduction for DCAN in Multivariate Logistic Regression}

For logistic regression, the continuous variables were transformed into grade variables according to the reference value. Multivariate logistic regression analysis was performed with DCAN as dependent variables and the amplitude/CV of motor nerve for the median, ulnar, posterior tibial, and peroneal nerves as independent variables. Similarly, multivariate logistic regression analysis was performed with DCAN as dependent variables and the amplitude/CV of sensory nerve for the median, ulnar, and sural nerves as independent variables. As shown in Table 3, a lower amplitude of motor fiber of the peroneal nerve was found to demonstrate an increased risk for DCAN (OR 2.77, 95\% CI 1.20-6.44, $P<0.05$ ).

TABLE 2 | Nerve conduction of patients with T2DM and DCAN versus those without DCAN.

\begin{tabular}{llccl}
\hline & & DCAN+ $(\boldsymbol{n}=\mathbf{4 2})$ & DCAN- $(\boldsymbol{n}=\mathbf{9 2})$ & \multicolumn{1}{c}{$\boldsymbol{P}$} \\
\hline Motor nerve & Amp $(\mathrm{mV})$ & $7.96 \pm 2.66$ & $9.60 \pm 2.22$ & $<0.001^{\star \star}$ \\
& $\mathrm{CV}(\mathrm{m} / \mathrm{s})$ & $44.22 \pm 5.10$ & $46.62 \pm 3.96$ & $<0.05^{\star}$ \\
Sensory nerve & Amp $(\mathrm{mV})$ & $17.90 \pm 13.66$ & $26.24 \pm 11.92$ & $<0.001^{\star \star}$ \\
& CV $(\mathrm{m} / \mathrm{s})$ & $48.13 \pm 7.27$ & $51.16 \pm 6.23$ & $<0.05^{\star}$ \\
\hline
\end{tabular}

Data were presented as mean $\pm S D$. Differences between the groups were analyzed using unpaired-sample t test.

Amp, Amplitude; CV, conduction velocity.

${ }^{*} P<0.05,{ }^{*} P<0.001$.

\section{SSR Value in DCAN+ and DCAN- Groups}

The SSR amplitude of the upper limb $[0.99(0.16-2.70) \mathrm{mV} v s$. $2.18(1.19-3.48) \mathrm{mV}, P<0.001]$ and lower limb $[0.44(0.13-1.38)$ $\mathrm{mV}$ vs. $1.17(0.73-1.97) \mathrm{mV}, P<0.001]$ were lower in the DCAN + group than in the DCAN- group. The SSR latency of the upper limb [1.49 (1.32-2.21) svs. $1.36(1.23-1.46) \mathrm{s}, P<0.05]$ and lower limb $[1.99(1.76-3.26)$ s vs. $1.79(1.62-1.93) \mathrm{s}, P<0.001]$ were higher in the DCAN+ group than in the DCANgroup (Table 4).

\section{ROC Analysis of SSR to Predict DCAN in Patients With T2DM}

ROC analysis revealed that the cutoff points of upper limb amplitude and lower limb amplitude, which maximized both the sensitivity and specificity to indicate DCAN, were $1.40 \mathrm{mV}$ (AUC $=0.70 ; 95 \%$ CI: 0.59-0.80; sensitivity, 61.9\%; specificity, $66.3 \%, P<0.001)$ and $0.85 \mathrm{mV}(\mathrm{AUC}=0.70$; 95\% CI: $0.60-0.80$; sensitivity, 66.7\%; specificity, 68.5\%, $P<0.001$ ), respectively. Similarly, the optimal cutoff points of upper limb latency and lower limb latency to indicate DCAN were $1.40 \mathrm{~s}$ (AUC $=0.67$; 95\% CI: 0.57-0.77; sensitivity, $61.9 \%$; specificity, $62 \%, P<0.05$ ) and $1.81 \mathrm{~s}$ (AUC $=0.66$; 95\% CI: 0.55-0.77; sensitivity, 69.0\%; specificity, 52.2\%, $P<0.05$ ), respectively (Figure 1).

TABLE 3 | Nerve conduction for DCAN in multivariate logistic regression.

\begin{tabular}{lcc}
\hline Variables & Odds ratio $(\mathbf{9 5 \%}$ Cl) & $P$ value \\
\hline Peroneal nerve M Amp & 1 (Ref.) & \\
$>2.6 \mathrm{mV}$ & $2.77(1.20-6.44)$ & $<0.05^{\star}$ \\
$<2.6 \mathrm{mV}$ &
\end{tabular}

M, motor nerve; Amp, Amplitude.

${ }^{*} P<0.05$. 
TABLE 4 | SSR of patients with T2DM and DCAN versus those without DCAN.

\begin{tabular}{|c|c|c|c|c|}
\hline & & DCAN+ $(n=42)$ & DCAN- $(n=92)$ & $P$ \\
\hline \multirow[t]{2}{*}{ Upper limb } & $\mathrm{Amp}(\mathrm{mV})$ & $0.99(0.16-2.70)$ & $2.18(1.19-3.48)$ & $<0.001^{\star \star}$ \\
\hline & Laten (s) & $1.49(1.32-2.21)$ & $1.36(1.23-1.46)$ & $<0.05^{\star}$ \\
\hline \multirow[t]{2}{*}{ Lower limb } & Amp (mV) & $0.44(0.13-1.38)$ & $1.17(0.73-1.97)$ & $<0.001^{\star \star}$ \\
\hline & Laten (s) & 1.99 (1.76-3.26) & 1.79 (1.62-1.93) & $<0.05^{\star}$ \\
\hline
\end{tabular}

Amp, Amplitude; Laten, latency.

${ }^{*} P<0.05,{ }^{*} P<0.001$.

\section{DISCUSSION}

DCAN results from damage to nerve fibers within the parasympathetic and sympathetic nervous systems. It is one of the leading causes of heart arrhythmias and is an independent risk factor for cardiovascular mortality among patients with diabetes. Early diagnosis and intervention in patients with T2DM can reduce DCAN progression. Recent guidelines strongly recommend screening for DCAN in patients with diabetes because the progression of cardiovascular denervation is partly reversible or can be slowed in the early stages of the disease (19).

In the present study, the overall prevalence of DCAN in a sample of patients with diabetes was $31.34 \%$. The prevalence reported in previous studies ranged from $25 \%$ to $75 \%$ in patients with T2DM (1, 20, 21). The significant variability between studies might be attributed to the lack of uniform diagnostic criteria and underdiagnosis due to inadequate screening $(1,22)$. In this study, DCAN was diagnosed using the Ewing test combined with HRV analysis to increase diagnostic specificity.

The association between diabetes duration and the development of DCAN has not been established. Interestingly, in the present study, older age was associated with DCAN but diabetes duration was not. A recent study also suggested that the duration of DM had no significant association with HRV measurements (23). However, a previous review reported that the prevalence of DCAN in patients with T2DM was associated with increased age and duration of diabetes (24). Pop-Busui et al. showed that DCAN was associated with poor glycemic control, increased age, and duration of disease, and diastolic blood pressure (25). Most likely, DCAN started in the early stages of diabetes (26). The discrepancy in results between studies might be due to inconsistent DCAN diagnostic criteria.

In the present study, the Valsalva ratio, E/I ratio, $30 \mathrm{~s} / 50 \mathrm{~s}$ ratio, and $\mathrm{OH}$ were different between DCAN+ and DCANgroups. Except for the rMSSD and PNN50, all the parameters of HRV analysis were lower in the DCAN+ group than in the

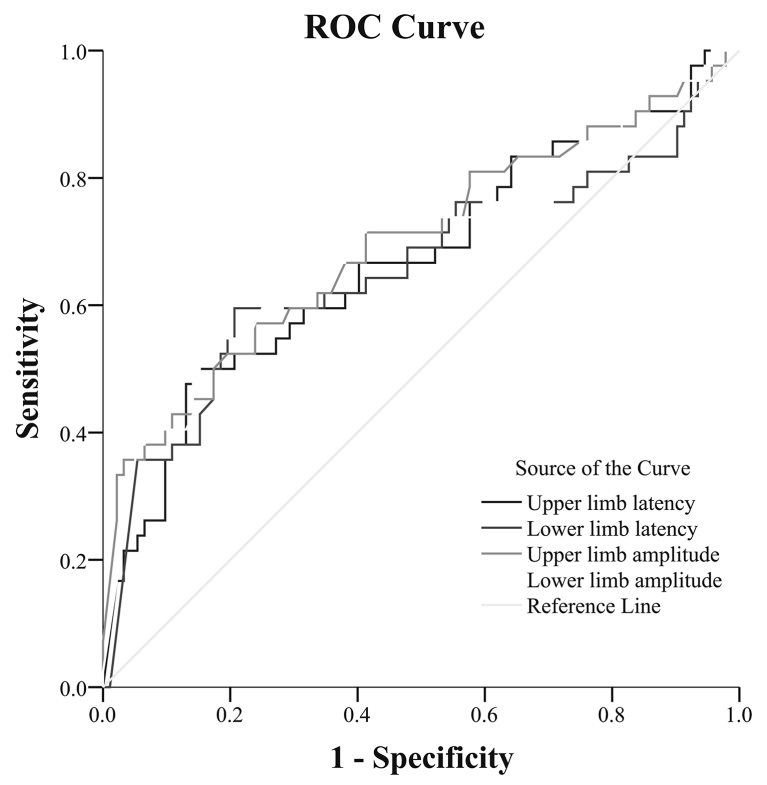

FIGURE 1 | Receiver-operating characteristic (ROC) analysis of SSR to predict DCAN in patients with T2DM [(upper limb amplitude: AUC = 0.70; 95\% Cl: 0.590.80; sensitivity, $61.9 \%$; specificity, $66.3 \%$, cut-point $1.40 \mathrm{mV}, P<0.001$ ) (lower limb amplitude: AUC = 0.70; $95 \%$ Cl: 0.60-0.80; sensitivity, 66.7\%; specificity, $68.5 \%$, cut-point $0.85 \mathrm{mV}, P<0.001$ ) (upper limb latency: AUC = 0.67; 95\% Cl: 0.57-0.77; sensitivity, $61.9 \%$; specificity, $62 \%$, cut-point $1.40 \mathrm{~s}, P<0.05)$ [lower limb latency: $A \cup C=0.66 ; 95 \%$ Cl: $0.55-0.77$; sensitivity, $69.0 \%$; specificity, $52.2 \%$, cut-point $1.81 \mathrm{~s}, P<0.05)]$. 
DCAN- group, indicating that both parasympathetic and sympathetic dysfunction existed during the development of CAN in patients with diabetes. This conclusion was consistent with that of multiple previous studies $(27,28)$. The results of the present study extended and confirmed these findings and suggested that T2DM was a metabolic disease responsible for cardiac autonomic neuropathy due to damage to both sympathetic and parasympathetic fibers.

The proportion of DPN was higher in the DCAN+ group than that in the DCAN - group. This finding was consistent with previous research (29). The amplitude and CV of the motor and sensory nerves were lower in the DCAN+ group than in the DCAN- group. Patients with severe DCAN had significantly reduced nerve CV and amplitude of peripheral nerves (30); it appeared that patients with diabetes and DCAN suffered more frequently from peripheral neuropathy compared with those without DCAN (31). A positive association existed between low HRV during deep breathing and large nerve fiber neuropathy, the latter documented with electrophysiology and clinical examination (32). Although some studies did not agree with these findings (33), a possible explanation was that CAN diagnosis was not based on a unanimous definition.

The present study found that DCAN was closely related to impaired peripheral nerve conduction. Moreover, NCS abnormality in the peroneal nerve (motor nerve) was more important in predicting DCAN. This was in line with previous studies. For example, parallel involvement of peripheral neuropathy and DCAN may principally affect the more vulnerable large nerve fibers (34). Parallel development of DCAN and peripheral neuropathy is thought to involve vulnerable large nerve fibers, with recent evidence pointing to an association between parasympathetic nervous system impairment and reduced peroneal motor nerve CV (35).

Peripheral NCS examine large-fiber sensory and motor nerve conduction. However, SSR is a simple, noninvasive method for evaluating small-fiber sudomotor function $(36,37)$. The sudomotor function is primarily mediated by the stimulation of the post sympathetic cholinergic fibers, which reflect the sweat nerve glands (unmyelinated C fiber) function or density (38). SSR latency measurements reflect the function of unmyelinated C fibers; SSR amplitude measurements reflect the density of spontaneously activable sweat glands (39). SSR appears to be useful for assessing autonomic neuropathy in patients with diabetes (40). In the present study, both the latency and the amplitude of SSR were different between the DCAN+ and DCAN- groups. The ROC analysis in the present study indicated that patients with T2DM should be alert for DCAN if SSR had upper limb amplitude lower than $1.40 \mathrm{mV}$, lower limb amplitude lower than $0.85 \mathrm{mV}$, upper limb latency longer than $1.40 \mathrm{~s}$, or lower limb latency longer than $1.81 \mathrm{~s}$.

In the present study, the results suggested that a lower amplitude of peroneal nerve motor fiber, as well as a lower amplitude and longer latency of SSR, were associated with increased risk of DCAN. Therefore, NCS and SSR can be used to predict and assist in DCAN diagnosis Nevertheless, the small sample size of the study is a limitation. Prospective studies with a larger sample size and long-term follow-up are required to confirm this conclusion.

\section{CONCLUSIONS}

The present study examined the role of NCS and sympathetic SSR in the evaluation of DCAN in diabetic patients. Consequently, this study revealed that a peroneal nerve (motor nerve) abnormality is crucial for predicting DCAN, wherein SSR may help. As DCAN is difficult to diagnose and predict, the data presented herein may be clinically useful.

\section{DATA AVAILABILITY STATEMENT}

The raw data supporting the conclusions of this article will be made available by the authors, without undue reservation.

\section{ETHICS STATEMENT}

The studies involving human participants were reviewed and approved by Ethics Committee of Nanfang Hospital, Southern Medical University. The patients/participants provided their written informed consent to participate in this study.

\section{AUTHOR CONTRIBUTIONS}

Each author has made an important scientific contribution to the study and is thoroughly familiar with the primary data. XL and CC carried out the clinical studies, participated in the statistical analysis and drafted the manuscript. YL, YP, ZC, and HH carried out the data acquisition, participated in the manuscript preparation and literature research. LX conceived of the study, and participated in its design and helped to review the manuscript. All authors contributed to the article and approved the submitted version.

\section{FUNDING}

This work was supported by Cultivating Plan Program for National Natural Science Foundation of Shenzhen Hospital, Southern Medical University under Grant NO. CNGZRJJPY202001.

\section{SUPPLEMENTARY MATERIAL}

The Supplementary Material for this article can be found online at: https://www.frontiersin.org/articles/10.3389/fendo.2021.709114/ full\#supplementary-material 


\section{REFERENCES}

1. Dimitropoulos G, Tahrani AA, Stevens MJ. Cardiac Autonomic Neuropathy in Patients With Diabetes Mellitus. World J Diabetes (2014) 5(1):17-39. doi: 10.4239/wjd.v5.i1.17

2. Maser RE, Lenhard JM, Decherney SG. Cardiovascular Autonomic Neuropathy: The Clinical Significance of Its Determination. Endocrinologist (2000) 10(1):27-33. doi: 10.1097/00019616-200010010-00006

3. Balcıoğlu AS, Müderrisoğlu H. Diabetes and Cardiac Autonomic Neuropathy: Clinical Manifestations, Cardiovascular Consequences, Diagnosis and Treatment. World J Diabetes (2015) 6(1):80-91. doi: 10.4239/wjd.v6.i1.80

4. Boulton AJ, Vinik AI, Arezzo JC, Bril V, Feldman EL, Freeman R, et al. Diabetic Neuropathies: A Statement by the American Diabetes Association. Diabetes Care (2005) 28(4):956-62. doi: 10.2337/diacare.28.4.956

5. Lin K, Wei L, Huang Z, Zeng Q. Combination of Ewing Test, Heart Rate Variability, and Heart Rate Turbulence Analysis for Early Diagnosis of Diabetic Cardiac Autonomic Neuropathy. Medicine (2017) 96(45):e8296. doi: $10.1097 / \mathrm{md} .0000000000008296$

6. Cygankiewicz I, Zareba W. Heart Rate Variability. Handb Clin Neurol (2013) 117:379-93. doi: 10.1016/b978-0-444-53491-0.00031-6

7. Mathias CJ. Autonomic Diseases: Clinical Features and Laboratory Evaluation. J Neurol Neurosurg Psychiatry (2003) 74Suppl 3(Suppl 3):iii3141. doi: 10.1136/jnnp.74.suppl_3.iii31

8. Ziegler D. Diabetic Cardiovascular Autonomic Neuropathy: Prognosis, Diagnosis and Treatment. Diabetes Metab Rev (1994) 10(4):339-83. doi: 10.1002/dmr.5610100403

9. Mala S, Potockova V, Hoskovcova L, Pithova P, Brabec M, Kulhankova J, et al. Cardiac Autonomic Neuropathy May Play a Role in Pathogenesis of Atherosclerosis in Type 1 Diabetes Mellitus. Diabetes Res Clin Pract (2017) 134:139-44. doi: 10.1016/j.diabres.2017.10.002

10. Tesfaye S, Boulton AJ, Dyck PJ, Freeman R, Horowitz M, Kempler P, et al. Diabetic Neuropathies: Update on Definitions, Diagnostic Criteria, Estimation of Severity, and Treatments. Diabetes Care (2010) 33(10):228593. doi: $10.2337 / \mathrm{dc} 10-1303$

11. Braffett BH, Gubitosi-Klug RA, Albers JW, Feldman EL, Martin CL, White $\mathrm{NH}$, et al. Risk Factors for Diabetic Peripheral Neuropathy and Cardiovascular Autonomic Neuropathy in the Diabetes Control and Complications Trial/Epidemiology of Diabetes Interventions and Complications (DCCT/EDIC) Study. Diabetes (2020) 69(5):1000-10. doi: $10.2337 / \mathrm{db} 19-1046$

12. Spallone V, Ziegler D, Freeman R, Bernardi L, Frontoni S, Pop-Busui R, et al. Cardiovascular Autonomic Neuropathy in Diabetes: Clinical Impact, Assessment, Diagnosis, and Management. Diabetes Metab Res Rev (2011) 27(7):639-53. doi: 10.1002/dmrr.1239

13. Pafili K, Trypsianis G, Papazoglou D, Maltezos E, Papanas N. Simplified Diagnosis of Cardiovascular Autonomic Neuropathy in Type 2 Diabetes Using Ewing's Battery. Rev Diabetic Stud RDS (2015) 12(1-2):213-9. doi: 10.1900/rds.2015.12.213

14. Pafili K, Trypsianis G, Papazoglou D, Maltezos E, Papanas N. Cardiovascular Autonomic Neuropathy and Distal Symmetric Sensorimotor Polyneuropathy: These Two Diabetic Microvascular Complications Do Not Invariably Co-Exist. Curr Vasc Pharmacol (2020) 18(1):50-6. doi: 10.2174/ 1570161116666180829120101

15. Heart Rate Variability: Standards of Measurement, Physiological Interpretation and Clinical Use. Task Force of the European Society of Cardiology and the North American Society of Pacing and Electrophysiology. Circulation (1996) 93(5):1043-65.

16. Metelka R. Heart Rate Variability-Current Diagnosis of the Cardiac Autonomic Neuropathy. A Rev Biomed Pap Med Fac Univ Palacky Olomouc Czech (2014) 158(3):327-38. doi: 10.5507/bp.2014.025

17. Shahani BT, Halperin JJ, Boulu P, Cohen J. Sympathetic Skin Response-a Method of Assessing Unmyelinated Axon Dysfunction in Peripheral Neuropathies. J Neurol Neurosurg Psychiatry (1984) 47(5):536-42. doi: 10.1136/jnnp.47.5.536

18. Ulas UH, Unlu E, Hamamcioglu K, Odabasi Z, Cakci A, Vural O. Dysautonomia in Fibromyalgia Syndrome: Sympathetic Skin Responses and RR Interval Analysis. Rheumatol Int (2006) 26(5):383-7. doi: 10.1007/s00296005-0007-1
19. Sztanek F, Jebelovs.zki É, Gaszner B, Zrínyi M, Páll D, Kempler P, et al. [Diagnosis of Diabetic Cardiac Autonomic Neuropathy]. Orvosi Hetilap (2019) 160(35):1366-75. doi: 10.1556/650.2019.31491

20. Agashe S, Petak S. Cardiac Autonomic Neuropathy in Diabetes Mellitus. Methodist DeBakey Cardiovasc J (2018) 14(4):251-6. doi: 10.14797/mdcj-144-251

21. Vinik AI, Ziegler D. Diabetic Cardiovascular Autonomic Neuropathy. Circulation (2007) 115(3):387-97. doi: 10.1161/circulationaha.106.634949

22. Ziegler D, Gries FA, Spüler M, Lessmann F. The Epidemiology of Diabetic Neuropathy. Diabetic Cardiovascular Autonomic Neuropathy Multicenter Study Group. J Diabetes Complications (1992) 6(1):49-57. doi: 10.1016/10568727(92)90049-q

23. Khandoker AH, Al-Angari HM, Khalaf K, Lee S, Almahmeed W, Al Safar HS, et al. Association of Diabetes Related Complications With Heart Rate Variability Among a Diabetic Population in the UAE. PloS One (2017) 12 (1):e0168584. doi: 10.1371/journal.pone.0168584

24. Vinik AI, Erbas T, Casellini CM. Diabetic Cardiac Autonomic Neuropathy, Inflammation and Cardiovascular Disease. J Diabetes Invest (2013) 4(1):4-18. doi: $10.1111 /$ jdi.12042

25. Pop-Busui R. Cardiac Autonomic Neuropathy in Diabetes: A Clinical Perspective. Diabetes Care (2010) 33(2):434-41. doi: 10.2337/dc09-1294

26. Fakhrzadeh H, Yamini-Sharif A, Sharifi F, Tajalizadekhoob Y, Mirarefin M, Mohammadzadeh M, et al. Cardiac Autonomic Neuropathy Measured by Heart Rate Variability and Markers of Subclinical Atherosclerosis in Early Type 2 Diabetes. ISRN Endocrinol (2012) 2012:168264. doi: 10.5402/2012/168264

27. Benichou T, Pereira B, Mermillod M, Tauveron I, Pfabigan D, Maqdasy S, et al. Heart Rate Variability in Type 2 Diabetes Mellitus: A Systematic Review and Meta-Analysis. PloS One (2018) 13(4):e0195166. doi: 10.1371/ journal.pone.0195166

28. Oakley I, Emond L. Diabetic Cardiac Autonomic Neuropathy and Anesthetic Management: Review of the Literature. AANA J (2011) 79(6):473-9.

29. Kim SH, Lee KA, Jin HY, Baek HS, Park TS. Relationship Between the Korean Version Survey of the Autonomic Symptoms Score and Cardiac Autonomic Neuropathy Parameters in Patients With Diabetic Peripheral Neuropathy. Diabetes Metab J (2014) 38(5):349-55. doi: 10.4093/dmj.2014.38.5.349

30. Haq T, Ahmed T, Latif ZA, Sayeed MA, Ashrafuzzaman SM. Cardiac Autonomic Neuropathy in Patients With Type 2 Diabetes Mellitus Having Peripheral Neuropathy: A Cross-Sectional Study. Diabetes Metab Syndr (2019) 13(2):1523-8. doi: 10.1016/j.dsx.2019.03.015

31. Voulgari C, Psallas M, Kokkinos A, Argiana V, Katsilambros N, Tentolouris N. The Association Between Cardiac Autonomic Neuropathy With Metabolic and Other Factors in Subjects With Type 1 and Type 2 Diabetes. J Diabetes Complications (2011) 25(3):159-67. doi: 10.1016/j.jdiacomp.2010.06.001

32. Orlov S, Bril V, Orszag A, Perkins BA. Heart Rate Variability and Sensorimotor Polyneuropathy in Type 1 Diabetes. Diabetes Care (2012) 35 (4):809-16. doi: 10.2337/dc11-1652

33. Töyry JP, Partanen JV, Niskanen LK, Länsimies EA, Uusitupa MI. Divergent Development of Autonomic and Peripheral Somatic Neuropathies in NIDDM. Diabetologia (1997) 40(8):953-8. doi: 10.1007/s001250050773

34. Pafili K, Trypsianis G, Papazoglou D, Maltezos E, Papanas N. Correlation of Cardiac Autonomic Neuropathy With Small and Large Peripheral Nerve Function in Type 2 Diabetes Mellitus. Diabetes Res Clin Pract (2019) 156:107844. doi: 10.1016/j.diabres.2019.107844

35. Lange-Maia BS, Newman AB, Jakicic JM, Cauley JA, Boudreau RM, Schwartz AV, et al. Relationship Between Sensorimotor Peripheral Nerve Function and Indicators of Cardiovascular Autonomic Function in Older Adults From the Health, Aging and Body Composition Study. Exp Gerontol (2017) 96:38-45. doi: 10.1016/j.exger.2017.04.007

36. Gerawarapong C. Association of Peripheral Autonomic Neuropathy and Sympathetic Skin Response in the Patients With Diabetic Polyneuropathy: A Pilot Study in Thailand. J Med Assoc Thailand = Chotmaihet Thangphaet (2015) 98(12):1222-30.

37. Hoitsma E, Reulen JP, de Baets M, Drent M, Spaans F, Faber CG. Small Fiber Neuropathy: A Common and Important Clinical Disorder. J Neurol Sci (2004) 227(1):119-30. doi: 10.1016/j.jns.2004.08.012

38. Stanley EF. Single Calcium Channels and Acetylcholine Release at a Presynaptic Nerve Terminal. Neuron (1993) 11(6):1007-11. doi: 10.1016/ 0896-6273(93)90214-c 
39. Levy DM, Reid G, Rowley DA, Abraham RR. Quantitative Measures of Sympathetic Skin Response in Diabetes: Relation to Sudomotor and Neurological Function. J Neurol Neurosurg Psychiatry (1992) 55(10):902-8. doi: 10.1136/jnnp.55.10.902

40. Spitzer A, Lang E, Birklein F, Claus D, Neundörfer B. Cardiac Autonomic Involvement and Peripheral Nerve Function in Patients With Diabetic Neuropathy. Funct Neurol (1997) 12(3-4):115-22.

Conflict of Interest: The authors declare that the research was conducted in the absence of any commercial or financial relationships that could be construed as a potential conflict of interest.
Publisher's Note: All claims expressed in this article are solely those of the authors and do not necessarily represent those of their affiliated organizations, or those of the publisher, the editors and the reviewers. Any product that may be evaluated in this article, or claim that may be made by its manufacturer, is not guaranteed or endorsed by the publisher.

Copyright (c) 2021 Lin, Chen, Liu, Peng, Chen, Huang and Xu. This is an open-access article distributed under the terms of the Creative Commons Attribution License (CC BY). The use, distribution or reproduction in other forums is permitted, provided the original author(s) and the copyright owner(s) are credited and that the original publication in this journal is cited, in accordance with accepted academic practice. No use, distribution or reproduction is permitted which does not comply with these terms. 